\title{
ON THE REMAINDER IN THE APPROXIMATE EVALUATION OF THE PROBABILITY IN THE SYMMETRICAL CASE OF JAMES BERNOULLI'S THEOREM*
}

BY C. D. OLDS

1. Introduction. In this paper we consider the symmetrical case of James Bernoulli's theorem in the theory of probability. We let $m$ represent the number of successes of an event in a series of $n$ independent trials with constant probability $p=$ $1-q=1 / 2$ for the success of each trial. Then we seek the probability $P$ of the inequality

$$
\left|m-\frac{n}{2}\right| \leqq \epsilon
$$

where $\epsilon$ is a given arbitrary positive number. The probability $P$ is usually given by an approximate formula without mention of the error term or remainder involved. $\dagger$ In 1926, D. Mirimanoff $\ddagger$ discussed this error term and gave results which are similar, but not as free from restrictions as those obtained here by entirely different methods. $\S$

2. The Exact Expression for $P$. Let $T_{m}$ represent the probability of $m$ successes in the $n$ trials and consider its generating function

$$
\sum_{m=0}^{n} T_{m} t^{m}
$$

where $t$ is an arbitrary variable. It has been shown $\|$ that

* Presented to the Society, April 3, 1937.

$\dagger$ See, for example, I. Todhunter, $A$ History of the Mathematical Theory of Probability, 1865, pp. 548-553.

$\ddagger$ D. Mirimanoff, Le jeu de pile ou face et les formules de Laplace et de $J$. Eggenberger, Commentarii Mathematici Helvetici, vol. 2 (1926), pp. 133-168.

$\S$ The author wishes to acknowledge the assistance rendered him by Professor J. V. Uspensky.

$\|$ For this and similar results see A. A. Markoff, Wahrscheinlichkeitsrechnung, 1912, pp. 18-44. 


$$
f(t)=\sum_{m=0}^{n} T_{m} t^{m}=\left(\frac{1}{2}\right)^{n}(t+1)^{n} .
$$

In this last expression we set $t=e^{i \phi}$, multiply through by $e^{-i m \phi}$, and then integrate between the limits $-\pi$ and $\pi$; we find

$$
T_{m}=\frac{1}{2 \pi} \int_{-\pi}^{\pi} f\left(e^{i \phi}\right) e^{-i m \phi} d \phi
$$

since

$$
\int_{-\pi}^{\pi} e^{(m-n) i \phi} d \phi= \begin{cases}0, & m \neq n, \\ 2 \pi, & m=n .\end{cases}
$$

Now let $\epsilon=-\frac{1}{2}+(n / 4)^{1 / 2} \zeta$ and express the inequality (1) in the form

$$
l_{1} \leqq m \leqq l_{2},
$$

where $l_{1}$ and $l_{2}$ are integers. Then the probability $P$ has the exact expression

$$
\begin{aligned}
P=\sum_{m=l_{1}}^{l_{2}} T_{m} & =\sum_{m=l_{1}}^{l_{2}} \frac{1}{2 \pi} \int_{-\pi}^{\pi}\left(\frac{1}{2}\right)^{n}\left(e^{i \phi}+1\right)^{n} e^{-i m \phi} d \phi \\
& =\frac{1}{2 \pi} \int_{-\pi}^{\pi}\left(\cos \frac{\phi}{2}\right)^{n} e^{i n \phi / 2} \sum_{m=l_{1}}^{l_{2}} e^{-i m \phi} d \phi
\end{aligned}
$$

Using the known identity

$$
\sum_{m=l_{1}}^{l_{2}} e^{-i m \phi}=\frac{i}{2 \sin \frac{\phi}{2}}\left\{e^{-i\left(l_{2}+1 / 2\right) \phi}-e^{-i\left(l_{1}-1 / 2\right) \phi}\right\},
$$

and substituting the values of $l_{1}$ and $l_{2}$, we find that

$$
P=\frac{1}{\pi} \int_{0}^{\pi}\left(\cos \frac{\phi}{2}\right)^{n} \frac{\sin \left(\frac{1}{2} \zeta n^{1 / 2} \phi\right)}{\sin \frac{\phi}{2}} d \phi .
$$

3. Three Lemmas. Let $\lambda$ be an arbitrary number such that $0<\lambda<\pi$. We use the expansion*

* L. L. Smail, Elements of the Theory of Infinite Processes, 1923, p. 245. 
$-\log \cos x=\left(2^{2}-1\right) \frac{2}{2 !} B_{1} x^{2}+\frac{1}{2}\left(2^{4}-1\right) \frac{2^{3}}{4 !} B_{2} x^{4}$

$$
+\frac{1}{3}\left(2^{6}-1\right) \frac{2^{5}}{6 !} B_{3} x^{6}+\cdots
$$

where $B_{1}, B_{2}, B_{3}, \cdots$ are the Bernoullian numbers. Consequently all the coefficients in this expansion are positive. Hence, we can deduce

$$
\begin{aligned}
& -\log \cos \frac{\phi}{2}=\frac{\phi^{2}}{8}+M \phi^{4}, \\
& -\log \cos \frac{\phi}{2}=\frac{\phi^{2}}{8}+\frac{\phi^{4}}{192}+N \phi^{6},
\end{aligned}
$$

where

$$
\begin{aligned}
& 0<M \leqq \lambda^{-4}\left(\log \sec \frac{\lambda}{2}-\frac{\lambda^{2}}{8}\right)=a, \\
& 0<N \leqq \lambda^{-6}\left(\log \sec \frac{\lambda}{2}-\frac{\lambda^{2}}{8}-\frac{\lambda^{4}}{192}\right)=b,
\end{aligned}
$$

provided $0<\phi \leqq \lambda$.

Likewise, from the expansion*

$$
\frac{\phi}{2 \sin \frac{\phi}{2}}=1+\sum_{k=1}^{\infty} \frac{\left(2^{2 k}-2\right)}{(2 k) !} B_{k}\left(\frac{\phi}{2}\right)^{2 k},
$$

where again all the coefficients are positive, we find that

where

$$
\frac{1}{\sin \frac{\phi}{2}}=\frac{2}{\phi}+\frac{\phi}{12}+L \phi^{3}
$$

$$
0<L \leqq \lambda^{-3}\left(\csc \frac{\lambda}{2}-\frac{2}{\lambda}-\frac{\lambda}{12}\right)=c,
$$

provided $0<\phi \leqq \lambda$.

* K. Knopp, Theory and Application of Infinite Series, 1928, p. 204. 
Using (3) we can easily show that

$$
\left|\left(\cos \frac{\phi}{2}\right)^{n}-e^{-\left(n \phi^{2}\right) / 8}\right|<n a \phi^{4} e^{-\left(n \phi^{2}\right) / 8}, \quad 0<\phi \leqq \lambda .
$$

From (4) we find that

$$
\left(\cos \frac{\phi}{2}\right)^{n}-e^{-\left(n \phi^{2}\right) / 8}\left(1-\frac{n \phi^{2}}{192}\right)=\left(-n N \phi^{6}+\frac{1}{2} \xi n^{2} M^{2} \phi^{8}\right) e^{-\left(n \phi^{2}\right) / 8},
$$

where $0<\xi<1$, and consequently

$$
\begin{aligned}
\left|\left(\cos \frac{\phi}{2}\right)^{n}-e^{-\left(n \phi^{2}\right) / 8}\left(1-\frac{n \phi^{2}}{192}\right)\right| \\
<\left(n b \phi^{6}+\frac{1}{2} n^{2} a^{2} \phi^{8}\right) e^{-\left(n \phi^{2}\right) / 8}, \quad 0<\phi \leqq \lambda .
\end{aligned}
$$

4. Application of (5), (6), and (7) to (2). Applying (5) to the integral on the right of (2), we have

$$
\begin{aligned}
P= & \frac{2}{\pi} \int_{0}^{\lambda}\left(\cos \frac{\phi}{2}\right)^{n} \frac{\sin \left(\frac{1}{2} \zeta n^{1 / 2} \phi\right)}{\phi} d \phi \\
& +\frac{1}{12 \pi} \int_{0}^{\lambda}\left(\cos \frac{\phi}{2}\right)^{n} \cdot \phi \cdot \sin \left(\frac{1}{2} \zeta n^{1 / 2} \phi\right) d \phi \\
& +\frac{1}{\pi} \int_{0}^{\lambda}\left(\cos \frac{\phi}{2}\right)^{n} \cdot L \cdot \phi^{3} \cdot \sin \left(\frac{1}{2} \zeta n^{1 / 2} \phi\right) d \phi \\
& +\frac{1}{\pi} \int_{\lambda}^{\pi}\left(\cos \frac{\phi}{2}\right)^{n} \cdot \frac{\sin \left(\frac{1}{2} \zeta n^{1 / 2} \phi\right)}{\sin \frac{\phi}{2}} d \phi .
\end{aligned}
$$

For brevity, we shall let the integrals in (8) be denoted by $I_{1}, I_{2}$, $I_{3}$, and $I_{4}$ respectively.

The inequality (7) shows that

(9) $\quad I_{1}=\frac{2}{\pi} \int_{0}^{\lambda} e^{-\left(n \phi^{2}\right) / 8}\left(1-\frac{n \phi^{4}}{192}\right) \frac{\sin \left(\frac{1}{2} \zeta n^{1 / 2} \phi\right)}{\phi} d \phi+\Delta_{(1)}$,

where

$$
\left|\Delta_{(1)}\right|<\frac{2}{\pi} \int_{0}^{\infty}\left(n b \phi^{5}+\frac{n^{2} a^{2}}{2} \phi^{7}\right) e^{-\left(n \phi^{2}\right) / 8} d \phi=\frac{1024 b+12288 a^{2}}{\pi n^{2}} .
$$


The integral in (9) splits into two integrals, the first of which gives

$$
\begin{aligned}
& \frac{2}{\pi} \int_{0}^{\lambda} e^{-(n \phi 2) / 8} \frac{\sin \left(\frac{1}{2} \zeta n^{1 / 2} \phi\right)}{\phi} d \phi \\
&=\frac{2}{\pi} \int_{0}^{\infty} e^{-(n \phi 2) / 8} \frac{\sin \left(\frac{1}{2} \zeta n^{1 / 2} \phi\right)}{\phi} d \phi+\Delta_{(2)},
\end{aligned}
$$

where

$$
\left|\Delta_{(2)}\right|<\frac{2}{\pi} \int_{\lambda}^{\infty} e^{-(n \phi 2) / 8} \frac{d \phi}{\phi}<\frac{8}{\pi \lambda^{2} n} e^{-\lambda 2 n / 8},
$$

since

$$
\int_{x}^{\infty} e^{-u^{2}} \frac{d u}{u}<\frac{e^{-x^{2}}}{2 x^{2}}, \quad x>0 .
$$

The integral in the right member of (10) is

$$
\begin{aligned}
\frac{2}{\pi} \int_{0}^{\infty} e^{-\left(n \phi^{2}\right) / 8} \frac{\sin \left(\frac{1}{2} \zeta n^{1 / 2} \phi\right)}{\phi} d \phi & =\frac{2}{\pi} \int_{0}^{\infty} e^{-\left(v^{2}\right) / 2} \frac{\sin (\zeta v)}{v} d v \\
& =\left(\frac{2}{\pi}\right)^{1 / 2} \int_{0}^{\zeta} e^{-\left(v^{2}\right) / 2} d v .
\end{aligned}
$$

We replace the second integral from (9) by

$$
\frac{2 n}{192 \pi} \int_{0}^{\infty} e^{-(n \phi 2) / 8} \phi^{3} \sin \left(\frac{1}{2} \zeta n^{1 / 2} \phi\right) d \phi+\Delta_{(3)},
$$

where

$$
\left|\Delta_{(3)}\right|<\frac{2 n}{192 \pi} \int_{\lambda}^{\infty} e^{-\left(n \phi^{2}\right) / 8} \phi^{3} d \phi=\frac{1}{3 \pi n}\left(1+\frac{n \lambda^{2}}{8}\right) e^{-(n \lambda 2) / 8} .
$$

We have

$$
\frac{2 n}{192 \pi} \int_{0}^{\infty} e^{-\left(n \phi^{2}\right) / 8} \phi^{3} \sin \left(\frac{1}{2} \zeta n^{1 / 2} \phi\right) d \phi=\frac{3 \zeta-\zeta^{3}}{6 n(2 \pi)^{1 / 2}} e^{-\left(\zeta^{2}\right) / 2},
$$

as is clear if we differentiate three times with respect to $\alpha$ the integral 


$$
\int_{0}^{\infty} e^{-\alpha u^{2}} \cos (\beta u) d u=\frac{1}{2}\left(\frac{\pi}{\alpha}\right)^{1 / 2} e^{-\beta /(4 \alpha)}, \quad \alpha>0,
$$

and make obvious substitutions.

5. The Integral $I_{2}$. If we apply the inequality (6) to $I_{2}$ we get

$$
I_{2}=\frac{1}{12 \pi} \int_{0}^{\lambda} e^{-(n \phi 2) / 8} \phi \sin \left(\frac{1}{2} \zeta n^{1 / 2} \phi\right) d \phi+\Delta_{(4)},
$$

where

$$
\left|\Delta_{(4)}\right|<\frac{n a}{12 \pi} \int_{0}^{\infty} e^{-(n \phi 2) / 8} \phi^{5} \cdot d \phi=\frac{128 a}{3 \pi n^{2}} .
$$

Also,

$$
\begin{aligned}
& \frac{1}{12 \pi} \int_{0}^{\lambda} e^{-(n \phi 2) / 8} \phi \sin \left(\frac{1}{2} \zeta n^{1 / 2} \phi\right) d \phi \\
&=\frac{1}{12 \pi} \int_{0}^{\infty} e^{-(n \phi 2) / 8} \phi \sin \left(\frac{1}{2} \zeta n^{1 / 2} \phi\right) d \phi+\Delta_{(5)}
\end{aligned}
$$

where

$$
\left|\Delta_{(5)}\right|<\frac{1}{12 \pi} \int_{\lambda}^{\infty} e^{-(n \phi 2) / 8} \phi d \phi=\frac{1}{3 \pi n} e^{-(n \lambda 2) / 8} .
$$

Using (11) again we find that

$$
\frac{1}{12 \pi} \int_{0}^{\infty} e^{-\left(n \phi^{2}\right) / 8} \phi \sin \left(\frac{1}{2} \zeta n^{1 / 2} \phi\right) d \phi=\frac{2 \zeta}{6 n(2 \pi)^{1 / 2}} e^{-\left(\zeta^{2}\right) / 2} .
$$

6. The Integrals $I_{3}$ and $I_{4}$. For the integral $I_{3}$ we have

$$
\left|I_{3}\right| \leqq \frac{1}{\pi} \int_{0}^{\lambda}\left(\cos \frac{\phi}{2}\right)^{n} \phi^{3} \cdot L \cdot d \phi<\frac{c}{\pi} \int_{0}^{\infty} e^{-\left(n \phi^{2}\right) / 8} \phi^{3} d \phi=\frac{32 c}{\pi n^{2}} .
$$

Likewise for $I_{4}$ we have

$$
\left|I_{4}\right| \leqq \frac{1}{\pi} \int_{\lambda}^{\pi}\left(\cos \frac{\phi}{2}\right)^{n} \cdot \frac{d \phi}{\sin \frac{\phi}{2}} \leqq \int_{\lambda}^{\pi}\left(\cos \frac{\phi}{2}\right)^{n} \frac{d \phi}{\phi},
$$

since, for $\phi \leqq \pi$, 


$$
\sin \frac{\phi}{2} \geqq \frac{\phi}{\pi}
$$

Also,

$$
\int_{\lambda}^{\pi}\left(\cos \frac{\phi}{2}\right)^{n} \frac{d \phi}{\phi}<\int_{\lambda}^{\infty} e^{-\left(n \phi^{2}\right) / 8} \frac{d \phi}{\phi}<\frac{4}{n \lambda^{2}} e^{-(n \lambda 2) / 8},
$$

which shows that

$$
\left|I_{4}\right|<\frac{4}{n \lambda^{2}} e^{-(n \lambda 2) / 8}
$$

7. The Remainder $\Delta$. Conclusion. Combining the above results we find that the probability $P$ of the inequality

$$
\left|m-\frac{n}{2}\right| \leqq-\frac{1}{2}+\zeta\left(\frac{n}{4}\right)^{1 / 2}
$$

is given by

$$
P=\left(\frac{2}{\pi}\right)^{1 / 2} \int_{0}^{\zeta} e^{-\left(v^{2}\right) / 2} d v+\frac{\zeta^{3}-\zeta}{6 n(2 \pi)^{1 / 2}} \cdot e^{-\left(\zeta^{2}\right) / 2}+\Delta,
$$

where for the remainder or error term $\Delta$ we have

$$
|\Delta|<\left[\frac{8}{n \lambda^{2} \pi}+\frac{4}{n \lambda^{2}}+\frac{1}{3 n \pi}\left(2+\frac{1}{8} n \lambda^{2}\right)\right] e^{-(n \lambda 2) / 8}+\frac{\omega}{n^{2} \pi},
$$

with

$$
\omega=\frac{128}{3} a+12288 a^{2}+1024 b+32 c .
$$

We now let $\lambda$ take on numerical values between 1 and 2 , and seek, corresponding to each, the smallest value of $n$ such that $|\Delta|<\left(n^{-2}\right) / 2$. It is found that, by taking $\lambda=1.8$, we have the inequality

$$
|\Delta|<\frac{1}{2 n^{2}}
$$

for $n \geqq 17$.

STANFORD UNIVERSITY 